\title{
PARHELIA: Particle Filter-Based Heart Rate Estimation from Photoplethysmographic Signals during Physical Exercise
}

\section{$\operatorname{AUTHOR}(\mathrm{S})$ :}

Fujita, Yuya; Hiromoto, Masayuki; Sato, Takashi

\section{CITATION:}

Fujita, Yuya ...[et al]. PARHELIA: Particle Filter-Based Heart Rate Estimation from Photoplethysmographic Signals during Physical Exercise. IEEE Transactions on Biomedical Engineering 2018, 65(1): 189-198

\section{ISSUE DATE:}

2018-01

URL:

http://hdl.handle.net/2433/223401

\section{RIGHT:}

(c) 2016 IEEE. Personal use of this material is permitted. Permission from IEEE must be obtained for all other users, including reprinting/ republishing this material for advertising or promotional purposes, creating new collective works for resale or redistribution to servers or lists, or reuse of any copyrighted components of this work in other works.; This is not the published version. Please cite only the published version.; この論文は出版社版でありません。引用の際には出 版社版をご確認ざ利用ください。 


\title{
PARHELIA: Particle Filter-Based Heart Rate Estimation from Photoplethysmographic Signals during Physical Exercise
}

\author{
Yuya Fujita, Non Member, Masayuki Hiromoto, Member, IEEE, and Takashi Sato, Member, IEEE
}

\begin{abstract}
The photoplethysmographic (PPG) signal is an important source of information for estimating heart rate (HR). However, the PPG signal could be strongly contaminated by the motion artifact (MA) of the subjects, making HR estimation a particularly difficult problem. In this paper, we propose PARHELIA, a PARticle filter-based algorithm for HEart rate estimation using photopLethysmographIc signAls. The proposed method employs a particle filter, and utilizes the simultaneously recorded acceleration signals from a wrist-type sensor, to keep track of multiple HR candidates. This achieves quick recovery from incorrect $H R$ estimations under the strong influence of the MA. Experimental results for a dataset of 12 subjects recorded during fast running showed that the average absolute estimation error was 1.17 beats per minute (BPM) whereas that of the bestknown conventional method, JOSS, is 1.28 BPM. Furthermore, the estimation time of PARHELIA is 20 times shorter than JOSS.
\end{abstract}

Index Terms-Heart rate estimation, motion artifact removal, particle filter, photoplethysmography (PPG).

\section{INTRODUCTION}

$\mathbf{H}$ EART rate (HR) monitoring is widely recognized as a useful measure for the exercisers to control their training load. There is a strong demand for HR monitoring devices that can be used during physical exercise. Various types of such devices are available in the market. They can be categorized based on the body part on which they are worn - chest, abdomen, wrist, etc. The devices worn on the chest or on the abdomen tend to obtain accurate HR estimations because they are tightly attached to the human body with a belt, making them resistant to measurement noise. However, wearing the tightened belt is uncomfortable for the users. Furthermore, chest-type and belt-type devices require an additional display device to indicate the current HR to the user. In contrast, wrist-type devices are, in general, much more comfortable to be worn compared to the other types of HR monitors, and are useful, because the display can be embedded into the device. In order to take advantage of these merits, we focus on developing an HR estimation method for the wrist-type HR monitors.

Y. Fujita, M. Hiromoto, and T. Sato are with Department of Communications and Computer Engineering, School of Informatics, Kyoto University, Yoshida-hon-machi, Sakyo, Kyoto, 606-8501 Japan. (correspondence e-mail: paper@easter.kuee.kyoto-u.ac.jp).

This work was supported in part by Japan Society for the Promotion of Science (JSPS) KAKENHI Grant Number 15K12700.

Copyright (C) 2016 IEEE. Personal use of this material is permitted However, permission to use this material for any other purposes must be obtained from the IEEE by sending an email to pubs-permissions@ieee.org.
Most of the wrist-type devices use photoplethysmographic (PPG) signals [1] to estimate the HR [2]. In typical PPG measurements, a device illuminates the skin and measures the changes in the intensity of the reflected light. The change in intensity corresponds to the volumetric change of the vessels under the skin; therefore, the HR can be estimated by capturing the periodicity of the PPG signal. However, PPG signals can be contaminated easily by motion artifacts (MAs), which makes HR estimation difficult.

Various methods to reduce the effect of MAs in PPG signals have been proposed. In [3], the noise in the PPG signal is removed by empirical mode decomposition (EMD). The original PPG signal is decomposed into intrinsic mode functions (IMF) components, which are then summed after MA reduction. In [4], the PPG signal is denoised using wavelet transform. The wavelet transform is first applied to the original signal, and subsequently, both the low-frequency and high-frequency components are removed by a threshold in the wavelet domain. Finally, the filtered data is transformed back to the time domain. Other methods include moving average filter [5], adaptive noise cancellation (ANC) [6], independent component analysis (ICA) [7], [8], Fourier series analysis [9], etc. These methods are used when the MAs are relatively small and the original shape of the PPG signal is not completely lost. A heuristic algorithm [10] is also proposed under the assumption that, during exercises, the MAs have higher harmonic contents than the PPG signal. It works well when there is a clear distinction between HR and MAs in the frequency domain.

There are other approaches that try to remove the MAs by using accelerometer signals, which include spectrum subtraction [11], [12], joint sparse spectrum reconstruction [13], singular spectrum analysis [2], [14], [15], adaptive filtering [16] and mixture of algorithms [17]. With the aid of an acceleration signal, strong and continuous MAs can be reduced. For example, in [11], [12], the MAs are reduced by subtracting the accelerometer spectrum from the PPG spectrum. More recently, JOSS [13] has been proposed to estimate the HR from PPG signals with strong MA contamination. JOSS consists of two parts: joint sparse spectrum reconstruction and spectral peak tracking. The joint sparse spectrum reconstruction is a novel approach that can be used to estimate the frequency spectrum instead of using ordinary methods such as fast Fourier transform (FFT). This unique methodology contributes to the high accuracy of JOSS in HR estimation.

However, there are some drawbacks in the JOSS method. It tracks only one candidate of the HR and uses the result of 
HR estimation of the previous time window for estimating the HR of the current time. Owing to this property, once the estimated HR deviates from the true HR, it is difficult to recover the true value from the incorrect estimate. Therefore, it is necessary for JOSS to employ a recovering algorithm, which is costly in terms of calculation time. Furthermore, JOSS needs an initialization stage that requires the subject to be at rest. Until the initialization is performed successfully, JOSS gives no estimation result.

Our study focuses on the HR estimation from the PPG signals that are recorded during physical exercise of changing intensity, in which the obtained PPG signals tend to be contaminated by continuous, random, and often large MAs. In this situation, the signal from the accelerometer is particularly helpful to identify the possible contamination of MAs, and this is why we decided to use accelerometer signals in our HR estimation.

In this paper, we propose a novel approach for HR estimation, PARHELIA. The proposed method utilizes a particle filter [18] to estimate the HR accurately under strong influence from MAs. The particle filter, which is one of the robust tracking methods using time series data, is suitable for the HR estimation, because the HR variation is limited within a small range in a short period. The existing works [2], [13] that are based on the same assumption have achieved good estimation results. In our work, unlike the existing methods, we take advantage of the particle filter that has a potential to recover from an incorrect estimation to the correct one, since an ensemble of the particles can always keep track of the multiple HR candidates. Tracking the multiple candidates ensures the robustness of our proposed HR estimation method. In addition, our method uses the acceleration signals in the weight calculation stage of the particle filter. By changing the weight of the particles according to the acceleration spectra, the noise caused by MAs can be sufficiently reduced.

The remainder of this paper is organized as follows. In Section II, the framework of the particle filter is described. In Section III, the proposed method of HR estimation, PARHELIA, is described. In Section IV, experimental results on the dataset are presented. Finally, we conclude this paper in Section V.

\section{PARTICLE Filter}

\section{A. Overview}

The particle filter can be used to solve non-linear tracking problems. By performing Bayesian estimation on a large number of particles, a probability density function (PDF) of a desired stochastic variable is approximated. The PDF is iteratively updated on the basis of observations, so that all the possible candidates can be tracked with the position of the particles.

Fig. 1 illustrates an example for a one-dimensional density estimation of the stochastic variable $x$ in a typical particle filter flow. The black circles represent the particles, and the curved lines express the density of particles. The purpose of the particle filter is to approximate the target distribution with the density of the particles. The salient feature of the particle

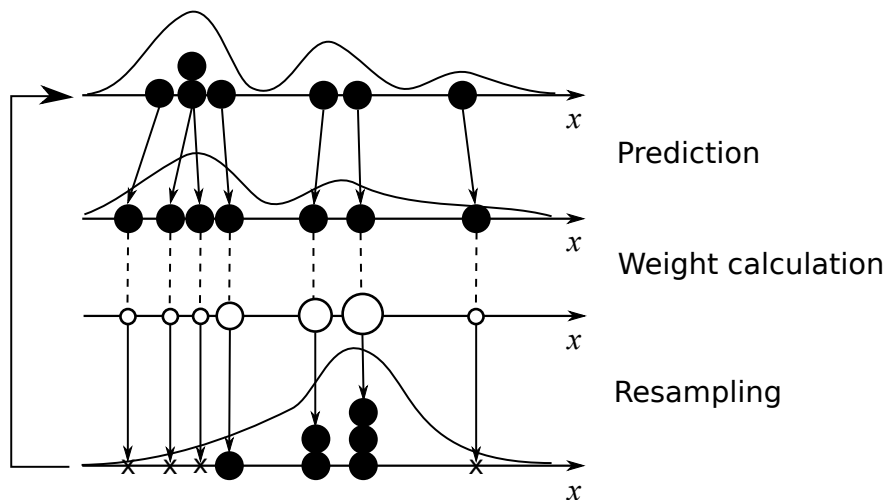

Fig. 1. Overview of a particle filter. In the prediction stage, each particle is moved according to (1). In the weight calculation stage, the weight of each particle is calculated by (2). In the resampling stage, the particles are extracted according to their weights. The above three stages are repeated.

filter is its ability to track multiple candidates; when there exist more than one candidate, the particle density has multiple peaks.

At the top of the figure, a distribution of particles at time $t-1$ is shown. In the next time step $t$, the state of the variable should change. In order to reflect the change, the particles are moved so that they fit the new state better. Typically, each movement is made using a random function. Based on the acquired signal, the fitness of each particle is evaluated and represented as the weight. Then, the particles are resampled according to the calculated weight. The particles that have very small weights will vanish, and the particles that have large weights will be copied many times, representing the distribution of the state.

In terms of the HR estimation, the stochastic variable $x$ represents HR at a particular time. The likelihood of the HR at the next time step is expressed by the density of particles based on the current position of the particles and the current observations of PPG and accelerometer signals. This update is realized by the following steps:

- Prediction: Each particle is moved to the next new position according to a prediction model, which defines the probabilistic behavior of the particles. For HR estimation, the prediction model should express the HR fluctuation caused by biorhythm and/or exercise. By moving all the particles to the new positions, the distribution of the particles changes to the likely distribution expected for the next time step.

- Weight calculation: The likelihood of the predicted particles is verified based on the current observation. If the predicted particle agrees well with the observation, a large weight is assigned to the particle; otherwise, a small weight is assigned. The size of the white circles in Fig. 1 represents the weights of the particles.

- Resampling: The particles are redistributed to express the weighted particles with the density of the particles. Thus, a new distribution of the particles representing the likelihood of the current state is obtained. The number of new particles is the same as the initial one, thus being ready as the input of the prediction step in the next 
iteration.

Kalman filter [19] is a well known method for solving tracking problems. In order for the Kalman filter to be applicable for a tracking problem, the system must be expressed by a linear model and the noise needs to follow the Gaussian distribution. At the same time, a particle filter does not need the above premise. In HR estimation, the HR may change suddenly and become heavily contaminated by MAs. This is why we use a particle filter instead of simple Kalman filters.

\section{B. Detailed Formulation}

In this subsection, we give a more formal derivation of the particle filter [19] and show how it is used in HR estimation.

A general non-linear/non-Gaussian state space model can be expressed as follows:

$$
\begin{aligned}
x_{t} & =f\left(x_{t-1}, v_{t}\right), \\
y_{t} & =h\left(x_{t}, w_{t}\right),
\end{aligned}
$$

where $f(\cdot)$ and $h(\cdot)$ represent non-linear functions, $x_{t}$ denotes a state of the system and follows the Markov process, $y_{t}$ is a measurement at time $t$, and $v_{t}$ and $w_{t}$ are the state and measurement noises, respectively. Let $y_{1: t}$ be the stacked vector of observations up to time $t$.

The important problem related to the state space model is estimating the unknown state $x_{t}$ using (1) and (2) when $y_{1: t}$ is given. For Bayesian estimation, this problem can be reviewed as a probabilistic inference process. The Bayesian estimation estimates a probability density function, $p\left(x_{t} \mid y_{1: t}\right)$, by using prediction and updating procedures as explained below. Here, $p\left(x_{t} \mid y_{1: t-1}\right)$ is the probability for the value $x_{t}$ to be true, from the observations $y_{1: t}$.

- Prediction: The PDF of $x$ is calculated at the current time $t$ by using (1) and all the observations until time $t-1$,

$$
p\left(x_{t} \mid y_{1: t-1}\right)=\int p\left(x_{t} \mid x_{t-1}\right) p\left(x_{t-1} \mid y_{1: t-1}\right) \mathrm{d} x_{t-1} .
$$

This calculation corresponds to the prediction step of the particle filter.

- Updating: The current observed value $y_{t}$ is used to update the prior PDF using Bayes' theorem,

$$
p\left(x_{t} \mid y_{1: t}\right)=\frac{p\left(y_{t} \mid x_{t}\right) p\left(x_{t} \mid y_{1: t-1}\right)}{p\left(y_{t} \mid y_{1: t-1}\right)},
$$

where the normalizing constant is

$$
p\left(y_{t} \mid y_{1: t-1}\right)=\int p\left(y_{t} \mid x_{t}\right) p\left(x_{t} \mid y_{1: t-1}\right) \mathrm{d} x_{t},
$$

which depends on the likelihood function $p\left(y_{t} \mid x_{t}\right)$ defined by (2). This part corresponds to the weight calculation and resampling steps in the particle filter.

In the above framework, the particle filter is defined as follows. Let $x_{t \mid t-1}$ and $x_{t \mid t}$ be the particles that express the probability density functions $p\left(x_{t} \mid y_{1: t-1}\right)$ and $p\left(x_{t} \mid y_{1: t}\right)$. Being $N$ the number of particles, these particle groups $X_{t \mid t-1}=\left\{x_{t \mid t-1}^{(i)}\right\}_{i=1}^{N}$ and $X_{t \mid t}=\left\{x_{t \mid t}^{(i)}\right\}_{i=1}^{N}$ are successively calculated using the following algorithm [18].

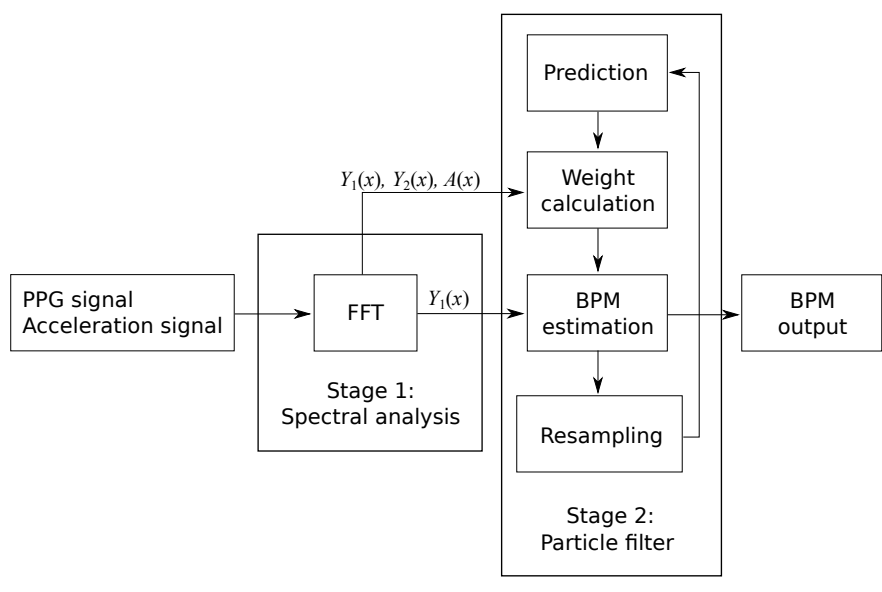

Fig. 2. Flowchart of the proposed method.

1) Generate particles $x_{0}^{(i)} \sim p\left(x_{0}\right)(i=1, \ldots, N)$, where " $\sim$ " represents the sampling from $p$.

2) For $t=1, \ldots, T$, carry out the following steps:

a) Repeat the following steps for each $i=1, \ldots, N$ :

i) Prediction: Generate $x_{t \mid t-1}^{(i)}$ from $x_{t-1 \mid t-1}^{(i)}$ using the prediction model, $x_{t \mid t-1}^{(i)} \sim p\left(x_{t} \mid x_{t-1}\right), \quad(i=1, \ldots, N)$.

ii) Weight calculation: Calculate the weight of the particles $w_{t}^{(i)}$ using the observation, $w_{t}^{(i)}=$ $p\left(y_{t} \mid x_{t \mid t-1}^{(i)}\right)$.

b) Calculate $W_{t}=\sum_{i=1}^{N} w_{t}^{(i)}$.

c) Resampling: Extract $x_{t \mid t-1}^{(i)}$ from $X_{t \mid t-1}=$ $\left\{x_{t \mid t-1}^{(i)}\right\}_{i=1}^{N}$ according to the weight $\hat{w}_{t}^{(i)}=$ $w_{t}^{(i)} / W_{t}$ by sampling with replacement.

\section{Proposed MEthod}

PARHELIA estimates the HR by using two PPG signals and 3-axis acceleration signals. The two PPG signals are independently recorded by the two adjacent PPG sensors equipped in a single wrist-type device. The accuracy of estimation is improved by using the two PPG sensors because one signal can compensate the other signal that might be contaminated by MAs, and vice versa. The acceleration signals are recorded by an accelerometer in the same device recording the PPG signals. They are used to reduce the influence of MAs.

PARHELIA consists of two stages: spectral analysis and particle filter. The particle filter is used to track multiple HR candidates. The flowchart of PARHELIA is shown in Fig. 2.

\section{A. Stage 1: Spectral Analysis}

The power spectra of two PPG signals and 3-axis acceleration signals are calculated using FFT with an order $N_{\mathrm{FFT}}$. $N_{\text {FFT }}$ is a parameter that decides the frequency resolution. In this case, the frequency resolution is expressed as

$$
\frac{F_{\mathrm{s}}}{N_{\mathrm{FFT}}} \times 60 \mathrm{BPM},
$$




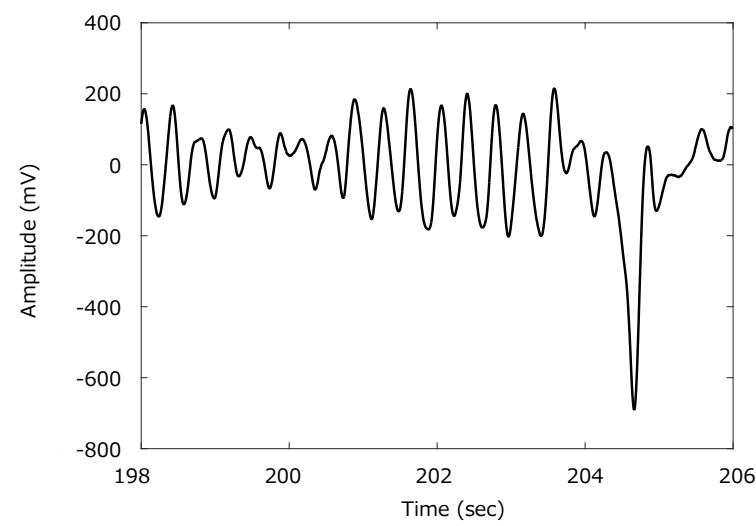

Fig. 3. An example of a raw PPG signal.

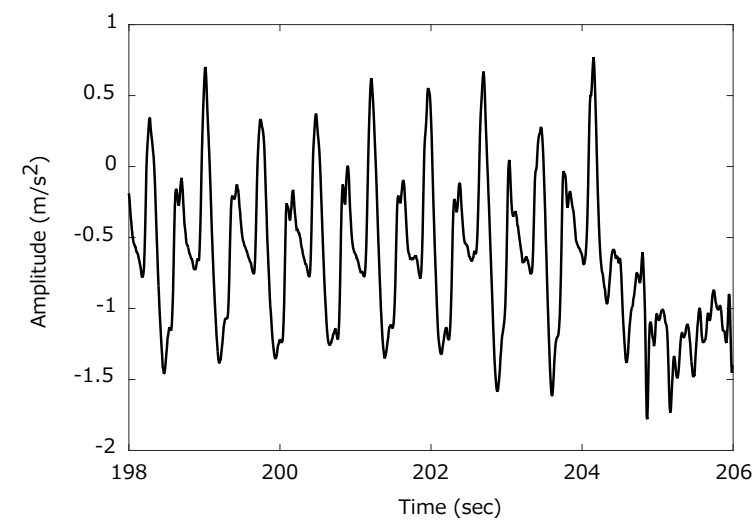

Fig. 5. An example of a raw acceleration signal (y-axis).

where $F_{\mathrm{s}}$ is a sampling frequency and BPM is beats per minute. The multiplication by 60 is a simple unit conversion from seconds to minutes. Figs. 3-6 show examples of the spectrum analysis for the PPG and accelerometer signals. Each power spectrum of the PPG signal is normalized by its maximum value. For an acceleration spectrum, an average of the three spectra of the 3 -axis acceleration signals are used, because the axis whose data corresponds to the MAs is unknown. In this work, the spectral analysis is performed using FFT, for its computational efficiency. However, it can be replaced by other spectrum analysis methods, such as the one proposed in [13].

\section{B. Stage 2: Particle Filter}

Let $x_{t}^{(i)}$ be the $i$-th particle that represents a value of BPM at time $t, Y_{1}(x)$ and $Y_{2}(x)$ are the two PPG spectra, and $A(x)$ is an acceleration spectrum. $x$ is distributed from 60 BPM to 210 BPM, which corresponds to a human's HR during physical exercises. The algorithm of the particle filter stage is described as follows.

1) Initialization: First, the distribution of particles at an initial state is decided. This corresponds to "1) Generate particles" in Section II-B. Since the HR is assumed to be relatively low but unknown at the beginning of the exercise,

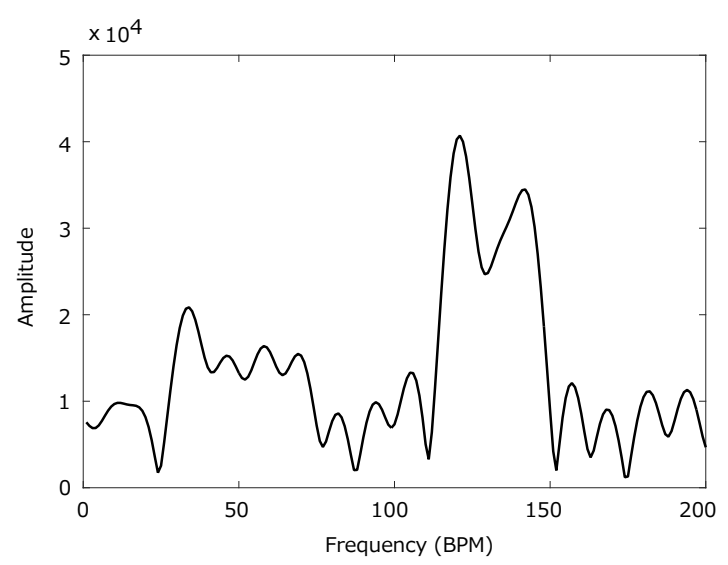

Fig. 4. FFT Spectra of the PPG signal in Fig. 3.

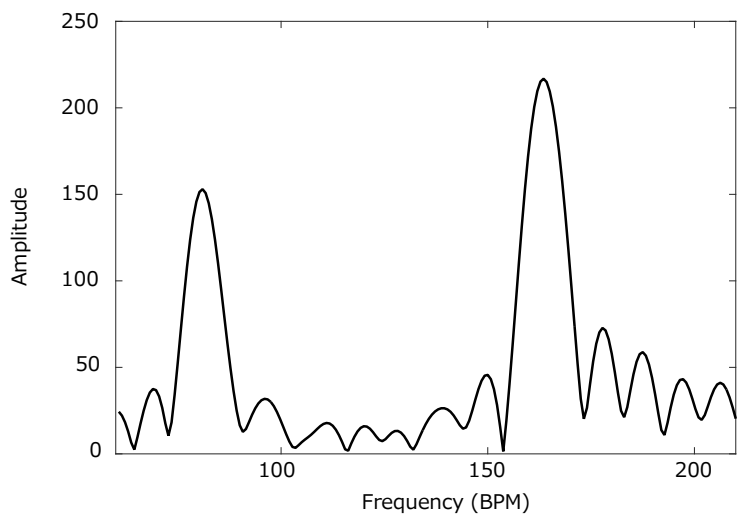

Fig. 6. FFT Spectra of the acceleration signal in Fig. 5.

we generate $X_{0}=\left\{x_{0}^{(i)}\right\}_{i=1}^{N}$ from $p\left(x_{0}\right)$, which is a uniform distribution in between $60 \mathrm{BPM}$ and $170 \mathrm{BPM}$. This range is used only for an initial distribution and the particles are allowed to move beyond that range during the iterations of the particle filter. The limit of the BPM range is discussed later in Section IV-D.

2) Prediction: As mentioned before, the prediction is performed based on (1). This corresponds to "2) a) i) Prediction" in Section II-B. Therefore, an appropriate prediction model for the HR change during exercise is required to achieve robust HR tracking. To construct an HR-aware prediction model, we used a dataset provided in [2] (see Section IV-A for details), which includes the ground truth of HR. These data enable us to plot the histogram for HR changes as shown in Fig. 7. In the figure, the horizontal axis shows the amount of BPM change, observed in the window of $2 \mathrm{~s}$.

In Fig. 7, the change in BPM is mostly in the range of \pm 6 BPM. Based on this observation, the prediction model of our particle filter is determined to use a normal distribution, whose mean is zero and standard deviation is 6 BPM. With these parameters, the maximum BPM change that may occur in $2 \mathrm{~s}$ can be tracked by the particle filter. These parameters, particularly the standard deviation, may be optimized better for each person or for each exercise; however, choosing relatively large values should work in most situations. Considering the 


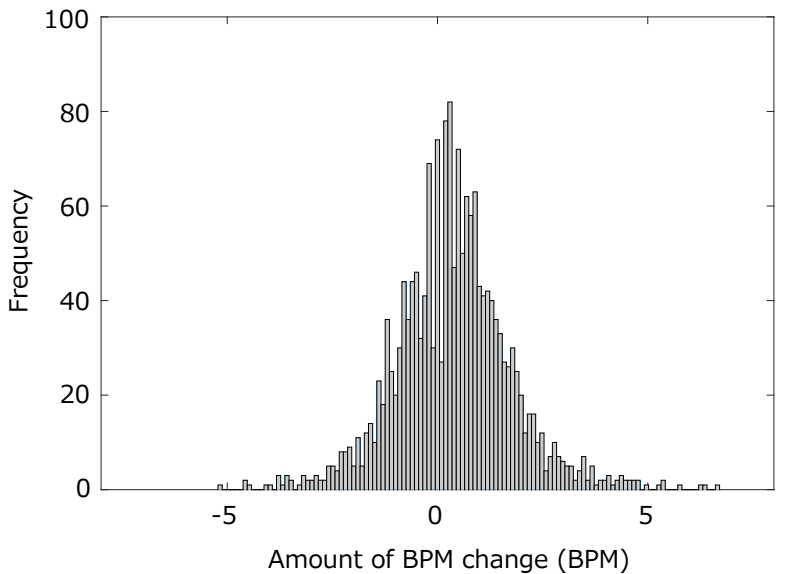

Fig. 7. Histogram for HR change in the dataset [2]. The difference in HR between two successive time windows and their corresponding frequencies are plotted.

bell-shape spread of the frequency components of the HR and by adopting a normal distribution with a sufficiently large standard deviation, the particle filter robustly keeps track of the change in HR even if a large and instantaneous change of HR is included in the data. In addition, even if the prediction fails to track the true peak of the HR, iterations of the particle filter catch up to obtain the peak of the HR.

Here, it should be noted that the prediction is performed by moving each particle according to a normal distribution with a standard deviation of 6 BPM. In most cases, when the particles spread in a wide area, a few time steps are sufficient to recover the correct HR estimation, even if the estimation in the previous time step is far from the correct value.

3) Weight Calculation: In this step, the weights of the particles are calculated using the PPG spectra $Y_{1}(x)$ and $Y_{2}(x)$, and the acceleration spectrum $A(x)$. This corresponds to "2) a) ii) weight calculation" in Section II-B. Here, $Y_{1}(x)$ and $Y_{2}(x)$ are contaminated by strong MAs as shown in Figs. 8 and 9. The spectrum amplitudes of $Y_{1}(x)$ and $Y_{2}(x)$, in the frequency range where that of the MA is large, need to be suppressed. For that purpose, $Y_{1}(x)$ and $Y_{2}(x)$ are modified as

$$
Y_{n}^{\prime}(x)=\left\{\begin{array}{ll}
Y_{n}(x) / k & \left(l_{1} \leq x \leq l_{2}, l_{3} \leq x \leq l_{4}\right) \\
Y_{n}(x) & \text { (otherwise) }
\end{array},\right.
$$

where $k$ is a weight coefficient, and $l_{1}$ and $l_{2}$ denote the left and the right ends of the mountain-like shape that are closest to the peak position of $A(x)$, as shown in Fig. 9. They are usually located in the range 60-135 BPM. Similarly, $l_{3}$ and $l_{4}$ define the second range of 135-210 BPM, as shown in Fig. 8.

According to (7), if the frequency of the MA is equal to or close to that of the HR, the spectrum amplitude of the HR might be reduced. However, it will not be an issue in reality. When the MA and HR almost completely overlap each other, the amplitude of that frequency shall become the tallest peak, which remains as the peak value even after the division of $k$. In addition, the maximum amplitude of the MA is close to that of the PPG, and therefore MAs do not completely overwhelm the PPG signal.

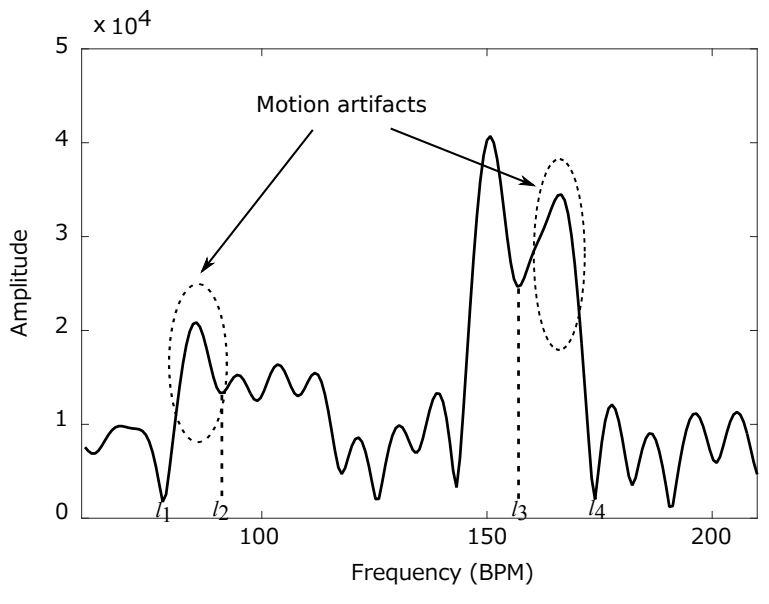

Fig. 8. Spectrum of PPG. It is contaminated by strong MA

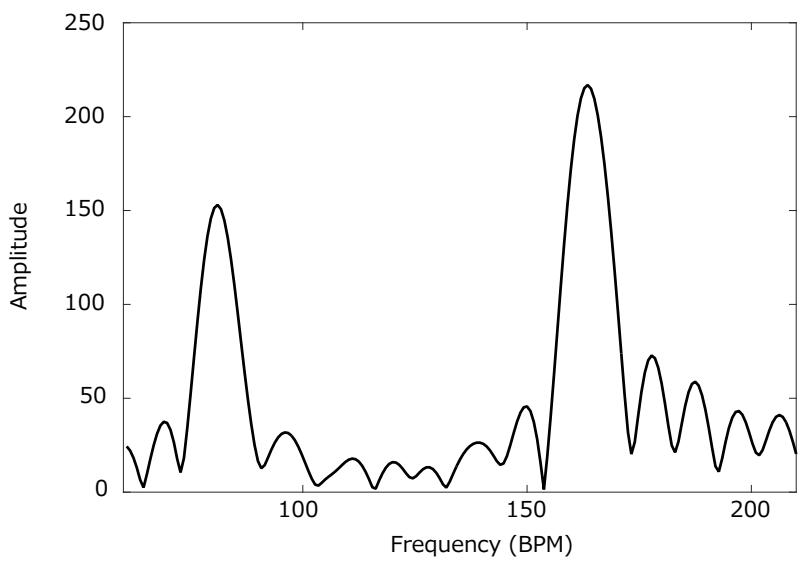

Fig. 9. Spectrum of acceleration signal.

$Y_{1}^{\prime}(x)$ and $Y_{2}^{\prime}(x)$ are the spectra where the MA effect is reduced. Using the two spectra, the weight of the particle is calculated by considering the two observations with equal importance;

$$
w_{t}\left(x_{t \mid t-1}^{(i)}\right)=Y_{1}^{\prime}\left(x_{t \mid t-1}^{(i)}\right)+Y_{2}^{\prime}\left(x_{t \mid t-1}^{(i)}\right),
$$

where $w_{t}\left(x_{t \mid t-1}^{(i)}\right)$ denotes the weight of particle $x_{t \mid t-1}^{(i)}$. They are then normalized so that the sum of all the weights becomes one.

4) BPM Estimation: In this step, the most likely BPM is decided using both the particle filter results and the PPG spectrum. The probability of the BPM $\beta$ to be the true HR, is estimated by

$$
p(\beta)=\sum_{x_{t \mid t-1}^{(i)}=\beta} w_{t}\left(x_{t \mid t-1}^{(i)}\right)
$$

Let $\beta_{\max }$ be the maximum point of $p(\beta)$, and it is defined as

$$
\beta_{\max }=\arg \underset{\beta}{\max }(p(\beta)) .
$$


Here, $\beta_{\max }$ is a point that is most likely to be the true HR according to the particle filter. The final estimation of the current BPM is then calculated by

$$
B P M_{\mathrm{cur}}=\left\{\begin{array}{ll}
x_{\text {peak } 1} & \left(\left|\beta_{\max }-x_{\text {peak } 1}\right|<d\right) \\
\beta_{\max } & \text { (otherwise) }
\end{array},\right.
$$

where $x_{\text {peak } 1}$ denotes the peak position of $Y_{1}(x)$, and $d$ is a distance threshold between $x_{\text {peak1 }}$ and $\beta_{\max }$. The reason why $x_{\text {peak } 1}$ is used instead of using $\beta_{\text {max }}$ directly is that the peak position of the current PPG spectrum often gives a more accurate BPM than that given by the particle filter does.

5) Resampling: This step corresponds to "2) c) Resampling" in Section II-B. There are several ways for resampling. For example, Kitagawa [20] proposed "random resampling," which is a common method for resampling. However, its computational time increases quadratically to the number of the particles. Therefore, it takes a long time to calculate the distribution of the particles, particularly when the number of particles is large. In order to reduce the computational cost, in this work, the particles are resampled according to the following procedure.

1) Let $p^{\prime}(\beta)=N \cdot p(\beta)$, so that the sum of $p^{\prime}(\beta)$ becomes the particle number $N$.

2) Round $p^{\prime}(\beta)$ up or down to the nearest decimal.

3) Generate particles so that the number of $\beta$ becomes $p^{\prime}(\beta)$.

This process is very fast since it has constant computational complexity. However, unlike random sampling, the number of the distributed particles may vary due to the rounding operation. If it is required to generate exactly $N$ samples, it is possible to perform additional random sampling to compensate the difference with $N$.

\section{EXPERIMENTAL RESULTS}

\section{A. Dataset}

In our experiments, we used a dataset from [2], which included two-channel PPG signals and 3-axis acceleration signals as the input of PARHELIA. They were all sampled at $125 \mathrm{~Hz}$. The PPG signals were recorded by two pulse oximeters with green LEDs, which were embedded in a wristband. The acceleration signals were recorded by a 3-axis accelerometer, which was also embedded in the wristband. In the dataset, a one-channel electrocardiogram (ECG) signal was available as the ground truth. The ECG signal was simultaneously recorded using wet ECG sensors firmly attached on the chest. The ECG signals were reliable enough to be used as the ground truth, because the electrical signals measured from the chest are less affected by MAs.

All the signals were recorded from 12 subjects having ages from 18 to 35 . The subjects either walked or ran during the data recording. The walking and running speeds were decided according to the following scenario: $1-2 \mathrm{~km} / \mathrm{h}$ for $0.5 \mathrm{~min}, 6-8 \mathrm{~km} / \mathrm{h}$ for $1 \mathrm{~min}, 12-15 \mathrm{~km} / \mathrm{h}$ for $1 \mathrm{~min}, 6-8 \mathrm{~km} / \mathrm{h}$ for $1 \mathrm{~min}, 12-15 \mathrm{~km} / \mathrm{h}$ for $1 \mathrm{~min}$, and $1-2 \mathrm{~km} / \mathrm{h}$ for $0.5 \mathrm{~min} .12$ $15 \mathrm{~km} / \mathrm{h}$ is considered to be fast enough for a normal human being; therefore, the data obtained by this protocol is suitable for evaluating the accuracy of HR estimation during typical running exercises.

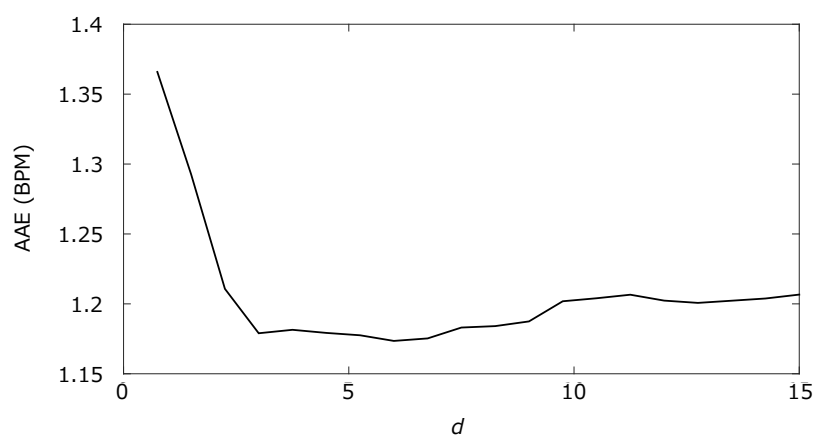

Fig. 10. AEE as a function of parameter $d . k$ is set to be 2.5.

\section{B. Settings}

The HR estimation is performed on a time window of $8 \mathrm{~s}$. The window slides with a step of $2 \mathrm{~s}$. In the spectral analysis stage, the order of FFT is set to $N_{\mathrm{FFT}}=10000$. This means that the resolution of BPM is $0.75 \mathrm{BPM}$. In the particle filter stage, the number of particle is set to $N=10000$ and the BPM estimation parameter is set to $d=4 \mathrm{BPM}$. Here, Fig. 10 shows the effect of choosing $d$ for the estimation error in absolute average error (AAE), when $k=2.5$. As seen in this figure, AAE rapidly grows for values of $d$ smaller than 3.5, meaning that when $\beta_{\max }$ and $x_{\text {peak }}$ in (11) are relatively close, $x_{\text {peak }}$, which gives the actual peak of the spectra, is a better choice to ensure the accuracy. When a relatively large value is chosen for $d$, it has an almost negligible effect on the estimation accuracy. The weight coefficient $k$ is an important parameter in the proposed method; therefore, we experimentally explore the appropriate value of $k$ from a range of 2.1 to 3 so that the average error becomes small, for all subjects. The proposed method is implemented using a MATLAB code.

\section{Performance Measurement}

We use the ground truth of HR given in the dataset [2] for the performance evaluation of the proposed method. Let $B P M_{\text {true }}(i)$ and $B P M_{\text {est }}(i)$ denote the ground truth of HR and the estimation of HR in the $i$-th time window, respectively. The estimation performance is evaluated by three indices. The first index is AAE, which is calculated by

$$
A A E=\frac{1}{W} \sum_{i=1}^{W}\left|B P M_{\text {est }}(i)-B P M_{\text {true }}(i)\right|,
$$

where $W$ is the total number of time windows.

The second index is Bland-Altman plot [21], in which the difference between the estimation and ground truth of HR is plotted against their average. The limit of agreement (LOA) [21], which is defined by $\left[\mu_{\mathrm{e}}-1.96 \sigma_{\mathrm{e}}, \mu_{\mathrm{e}}+\right.$ $\left.1.96 \sigma_{\mathrm{e}}\right]$, where $\mu_{\mathrm{e}}$ denotes the average of the difference and $\sigma_{\mathrm{e}}$ denotes the standard deviation of the difference, is calculated for evaluating the error distribution. Under the assumption that the error follows a Gaussian distribution, $95 \%$ of the errors are expected to be in this range. Most of the errors are expected to be in this range, therefore the LOA can be estimated to be the substantial upper and lower limits of the errors. If the LOA 


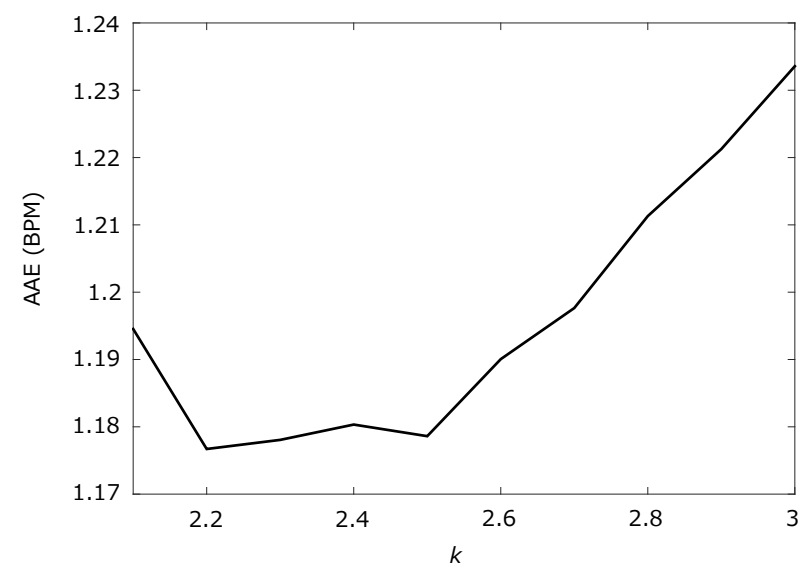

Fig. 11. AEE as a function of the variable $k$. AAE takes a small value around $k=2.2-2.5$.

becomes smaller, the maximal error should become smaller Thus, we use LOA for evaluating the accuracy of estimation.

The third index is Pearson correlation. Given two data rows $\left\{\left(x_{i}, y_{i}\right)\right\}(i=1,2, \ldots, n)$, Pearson correlation coefficient is calculated by

$$
r=\frac{\sum_{i=1}^{n}\left(x_{i}-\bar{x}\right)\left(y_{i}-\bar{y}\right)}{\sqrt{\sum_{i=1}^{n}\left(x_{i}-\bar{x}\right)^{2}} \sqrt{\sum_{i=1}^{n}\left(y_{i}-\bar{y}\right)^{2}}},
$$

where $\bar{x}$ and $\bar{y}$ are the arithmetic means of $x=\left\{x_{i}\right\}$ and $y=$ $\left\{y_{i}\right\}$, respectively. The more the coefficient becomes close to 1 , the linear dependence between the variables becomes more significant. In this work, the correlation between the groundtruth of HR and the estimated values are evaluated.

\section{Results and Discussion}

First, we explore the appropriate value of the particle-weight coefficient $k$ in (7). Here, $k$ is swept from 2.1 to 3 with an increment of 0.1 , and the respective AAE is compared. The result is shown in Fig. 11. We see that AAE becomes minimum when $k=2.2-2.5$. It should be noted that the AAE does not vary significantly, thus giving stable errors in a range between 1.17 BPM and 1.24 BPM, for the given range of $k$. The appropriate value of $k$ may change depending on the subject's health condition, noise intensity, etc. However, even in cases where the selection of $k$ is suboptimal, the estimation accuracy does not degrade significantly. Based on this analysis, 2.5 is chosen as the value of $k$ for the rest of the evaluation.

Table I lists the AAE for 12 subjects for the previous work, JOSS [13], and the proposed method, PARHELIA. It can be seen that, in most cases, PARHELIA achieves a better performance than JOSS. The average AAE of PARHELIA (60-210 BPM, 2ch PPG, w/o data trimming) is 1.17 BPM, whereas that of JOSS is $1.28 \mathrm{BPM}$. It means that PARHELIA can estimate HR more accurately than JOSS by $8.6 \%$. In the results of JOSS reported in [13], the results of the first few frames, until it successfully captures the accurate HR, are missing. It is because the initial recordings contain strong MA, probably due to the device adjustment after the recording system was turned ON, as mentioned in [13]. In JOSS, the

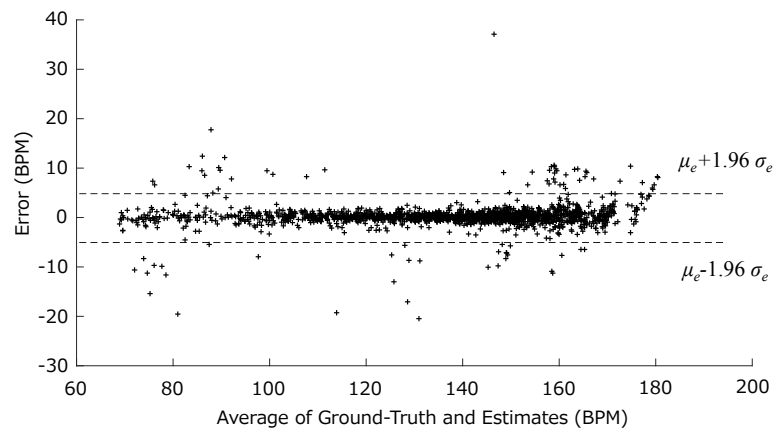

Fig. 12. Bland-Altman plot of the HR estimation by PARHELIA over 12 subjects. The LOA are $[-4.65,5.09]$ BPM.

calculated HR has to be used for the estimation of the next step, and therefore failing to capture the HR in the very first region of the data seems to be the reason for missing the estimation of the subsequent results. The data for subjects $\# 2$, \#3, \#4, \#8, \#10, and \#11 are missing. In order to make apple-to-apple comparison, additional experiments are added for PRAHELIA by removing the initial few frames for the subjects that have missing results in JOSS. In this condition, AAE of the proposed method further improved to 1.12 BPM.

In addition, we also evaluate the absolute average error of the proposed algorithm for 12 subjects by changing the range of particles, assuming that the range of the BPM is unavailable in advance. In this evaluation, the valid range of the particle movement is 40-210 BPM, while maintaining the number of particles. This result is also shown in Table I. The results in the case of a wider range (40-210 BPM) shows that PARHELIA obtains better estimations than the state of the art method, JOSS, although widening the particle distribution range slightly deteriorates the BPM estimation. Furthermore, we confirmed that the BPM range used in the initial stage estimation does not change the overall estimation result significantly. It is because particles quickly follow the changes of the HR by the particle filter iterations.

If we use the data of one of the two PPG sensors, the AAE for 12 subjects becomes worse from 1.17 to 1.58 , in the cases without data trimming. As shown in Table I, the AAE of all but one subjects degraded, showing that the estimation accuracy improves by using two PPG signals. However, with data trimming, the AAE of the single-PPG estimation only decreases from 1.12 to 1.26 , which means that PARHELIA with a single PPG still outperforms JOSS under the same experimental conditions.

Fig. 12 shows the Bland-Altman plot of the HR estimation for the 12 subjects. The LOA of PARHELIA were [-4.65, 5.09] BPM, whereas the LOA of JOSS were $[-5.94,5.41]$ BPM. The smaller range of the LOA of PARHELIA means that the possibility of large errors is less often than the case of JOSS; e.g., the probability that the estimation error of PARHELIA exceeds the LOA of JOSS is about $2.5 \%$, whereas that of JOSS is $5 \%$ according to the definition of LOA. When we perform the evaluation on the trimmed data using a single PPG signal, the LOA became [-5.07, 5.56]. 
TABLE I

ABSOLUTE AVERAGE ERROR FOR 12 SUBJECTS $^{1}$

\begin{tabular}{lc|c|cccccc}
\hline Method & & \multicolumn{7}{|c}{ PARHELIA } \\
\hline Settings & BPM range & - & $60-210$ & $60-210$ & $40-210$ & $60-210$ & $60-210$ & $40-210$ \\
& \# PPG ch. & 1 & 1 & 2 & 2 & 1 & 2 & 2 \\
& Data Trimming & Yes & Yes & Yes & Yes & No & No & No \\
\hline Subject No. & 1 & $\mathbf{1 . 3 3}$ & 2.21 & 1.74 & 1.72 & 2.18 & 1.82 & 1.73 \\
& 2 & 1.75 & $\mathbf{1 . 3 5}$ & $\mathbf{1 . 0 4}$ & $\mathbf{1 . 5 1}$ & 2.46 & $\mathbf{1 . 2 9}$ & 2.17 \\
& 3 & 1.47 & $\mathbf{1 . 0 2}$ & $\mathbf{0 . 8 0}$ & $\mathbf{0 . 7 1}$ & $\mathbf{0 . 8 6}$ & $\mathbf{0 . 8 0}$ & $\mathbf{0 . 7 5}$ \\
& 4 & 1.48 & $\mathbf{0 . 8 8}$ & $\mathbf{1 . 0 4}$ & $\mathbf{0 . 9 2}$ & $\mathbf{0 . 9 7}$ & $\mathbf{0 . 9 9}$ & $\mathbf{0 . 8 9}$ \\
& 5 & 1.69 & $\mathbf{0 . 6 3}$ & $\mathbf{0 . 6 5}$ & $\mathbf{0 . 6 6}$ & $\mathbf{0 . 6 6}$ & $\mathbf{0 . 6 5}$ & $\mathbf{0 . 6 5}$ \\
& 6 & 0.71 & $\mathbf{0 . 6 5}$ & $\mathbf{0 . 6 9}$ & $\mathbf{0 . 7 0}$ & $\mathbf{0 . 6 5}$ & $\mathbf{0 . 6 2}$ & $\mathbf{0 . 6 2}$ \\
& 7 & 0.56 & 0.64 & 0.64 & $\mathbf{0 . 5 2}$ & $\mathbf{0 . 5 3}$ & 0.62 & $\mathbf{0 . 5 1}$ \\
& 0.49 & $\mathbf{0 . 4 4}$ & $\mathbf{0 . 4 1}$ & $\mathbf{0 . 4 2}$ & $\mathbf{0 . 4 4}$ & $\mathbf{0 . 4 0}$ & $\mathbf{0 . 4 0}$ \\
& 9 & 3.81 & 3.88 & $\mathbf{3 . 1 2}$ & $\mathbf{3 . 2 2}$ & 4.89 & $\mathbf{3 . 6 2}$ & 3.90 \\
& 10 & $\mathbf{0 . 7 8}$ & 0.89 & 0.99 & 1.14 & 0.86 & 0.92 & 1.04 \\
& 11 & $\mathbf{1 . 0 4}$ & 1.47 & 1.22 & 1.18 & 3.42 & 1.24 & 1.22 \\
\hline
\end{tabular}

${ }^{1}$ The numbers in bold indicate better result when the error of each of the proposed method is compared to that of the state of the art method, JOSS

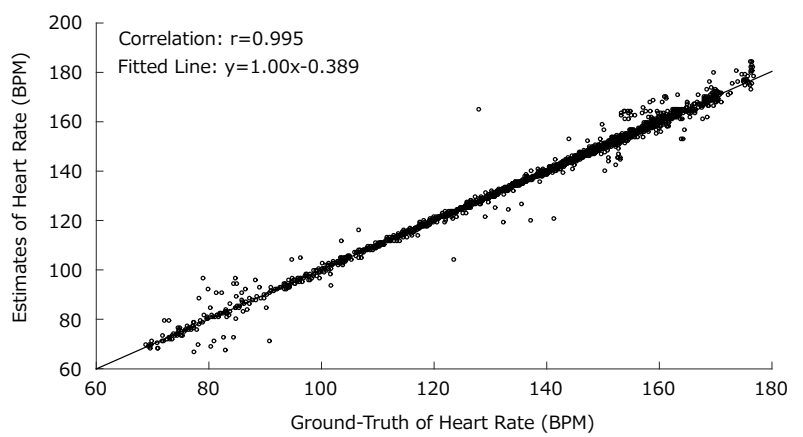

Fig. 13. Scatter plot between the ground-truth of HR and the estimations over 12 subjects. The fitted line was $y=1.00 x-0.389$, where $x$ indicates the ground truth of HR, and $y$ indicates the corresponding estimation. The Pearson correlation coefficient was 0.995 .

Fig. 13 shows a scatter plot between the ground truth and the estimation of HR over 12 subjects. The Pearson correlation coefficient of PARHELIA is 0.995 whereas that of the existing method is 0.993 . When we perform the evaluation on the trimmed data using a single PPG signal, the Pearson correlation became 0.994 .

To summarize, the three index values of performance for estimations by PARHELIA are all better than the existing method. This is largely due to the quick recovery property of PARHELIA, which enables it to recover faster than the existing method, even when unavoidable incorrect estimation occurs due to strong MA, etc. In addition, in PARHELIA, the frequency of giving incorrect estimates is less.

Fig. 14 shows the traces of the estimated HR by PARHELIA and JOSS for Subject \#4. The particle density in PARHELIA estimation at each time is also presented as a contour map. It can be seen that JOSS takes several seconds to recover from the incorrect estimation (approximately 30s), while PARHELIA quickly recovers from the incorrect estimations due to the tracking of multiple HR candidates with the aid of particles. As it can be seen from the contour map in Fig. 14, the particles are distributed in a wide range, covering the changes of the ground truth. Although many particles are concentrated

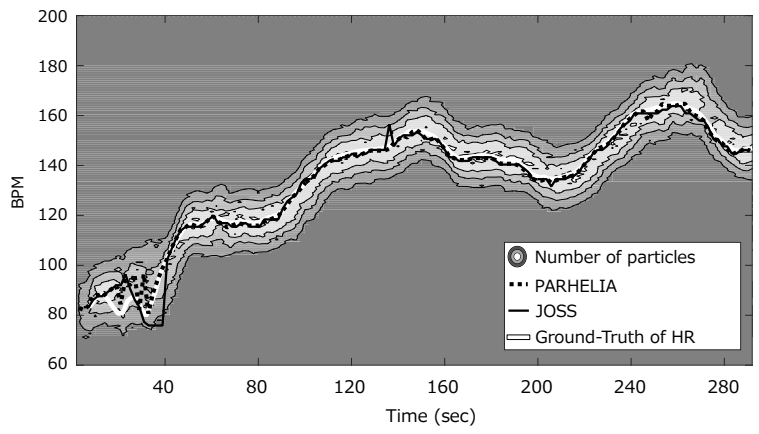

Fig. 14. The traces of the estimated HR by PARHELIA and JOSS. The number of particles in PARHELIA is also represented as a contour. It can be seen that PARHELIA quickly recovers from the incorrect estimation as it tracks multiple HR candidates.

close to the prior estimations of $\mathrm{HR}$, the estimation value can change by about $20 \mathrm{BPM}$ in one step, which makes it possible to recover than JOSS. In addition, the number of successive errors, which is both larger than 10 BPM and more than two time windows in all the subjects are 7 in PARHELIA and 24 in JOSS. From this result, it can be said that PARHELIA quickly recovers from incorrect estimations.

\section{E. Processing Time}

We compare the processing time of PARHELIA and JOSS. We implemented the spectral analysis part of JOSS by using the MATLAB code published in [22]. In general, JOSS takes a long time for its spectral analysis. JOSS takes about $0.6 \mathrm{~s}$ to estimate a single $\mathrm{HR}$ of a subject with $F_{\mathrm{s}}=25 \mathrm{~Hz}$ on a computer equipped with Intel Xeon X5570 running at $2.93 \mathrm{GHz}$ and MATLAB R2015a, whereas PARHELIA takes only $0.03 \mathrm{~s}$ with $F_{\mathrm{S}}=125 \mathrm{~Hz}$ on the same computer. PARHELIA estimates the HR about 20 times faster than JOSS. If PERHELIA is run on an embedded system with a slower processor, the processing speed may decrease. However, even if the embedded processor is 60 times slower than the Xeon processor, PERHELIA can 
TABLE II

PROCESSING TIME PROFILE OF A SINGLE STEP HR ESTIMATION (8 $\mathrm{S}$ WINDOW)

\begin{tabular}{lrc}
\hline & PARHELIA & JOSS \\
\hline Spectral analysis & $12 \mathrm{~ms}$ & $600 \mathrm{~ms}$ \\
Particle filter & $15 \mathrm{~ms}$ & \\
Prediction & $0.6 \mathrm{~ms}$ & \\
Weight calculation & $1.6 \mathrm{~ms}$ & \\
BPM estimation & $13 \mathrm{~ms}$ & \\
Resampling & $0.07 \mathrm{~ms}$ & \\
\hline Total & $27 \mathrm{~ms}$ & $600 \mathrm{~ms}+\alpha$ \\
\hline
\end{tabular}

still perform at the speed of a single HR estimation per $2 \mathrm{~s}$, which is sufficient for online and real-time estimations.

Table II shows a breakdown of the processing time for a single HR estimation by PARHELIA and JOSS. The most computationally intensive procedure for JOSS is the singular value decomposition (SVD) in spectral analysis. The SVD for dense matrix has a computational complexity of $\mathcal{O}\left(n^{2} m\right)$, where $n$ is the row size and $m$ is the column size of the matrix $(n<m)$. One of the computational bottlenecks in the proposed method is FFT, which has the complexity of $\mathcal{O}(n \log n)$. The process of particle filter does not involve computationally intensive matrix manipulations at all. Thus, the proposed method is much faster than JOSS.

\section{CONCLUSION}

In this paper, we proposed a novel method called PARHELIA, a particle filter-based HR estimation algorithm that uses PPG signals and simultaneously recorded acceleration signals using a wrist-type sensor. By applying the particle filter, multiple BPM candidates can be tracked, ensuring robustness to incorrect estimations. In addition, the acceleration signal is used in the weight calculation stage of the particle filter. By reducing the weight of the particles according to the acceleration spectrum, the noise caused by MAs is reduced. The experimental results for 12 subjects, though limited, showed that PARHELIA achieved $8.6 \%$ better estimation accuracy and 20 times faster performance than JOSS, which is a recent reference method in this field. Our future work includes adding more test cases on larger number of subjects under various exercises in order to more comprehensively evaluate the strength and weakness of various HR estimation methods.

\section{REFERENCES}

[1] J. Allen, "Photoplethysmography and its application in clinical physiological measurement," Physiol. Meas., vol. 28, no. 3, p. R1, 2007.

[2] Z. Zhang et al., "TROIKA: A general framework for heart rate monitoring using wrist-type photoplethysmographic signals during intensive physical exercise," IEEE Trans. Biomed. Eng., vol. 62, no. 2, pp. 522531,2015

[3] X. Sun et al., "Robust heart beat detection from photoplethysmography interlaced with motion artifacts based on empirical mode decomposition," in Proc. Int. Conf. Biomed. Health Inform. (BHI), 2012, pp. 775 778.

[4] M. Raghuram et al., "Evaluation of wavelets for reduction of motion artifacts in photoplethysmographic signals," in Proc. Int. Conf. Information Science, Signal Processing and their Applications (ISSPA), May 2010, pp. 460-463.

[5] H.-W. Lee et al., "The periodic moving average filter for removing motion artifacts from PPG signals," Int. J. Control Automation and Systems, vol. 5, no. 6, pp. 701-706, 2007.
6] R. Yousefi et al., "A motion-tolerant adaptive algorithm for wearable photoplethysmographic biosensors," IEEE J. Biomed. Health Inform., vol. 18, no. 2, pp. 670-681, 2014.

[7] B. S. Kim and S. K. Yoo, "Motion artifact reduction in photoplethysmography using independent component analysis," IEEE Trans. Biomed. Eng., vol. 53, no. 3, pp. 566-568, 2006.

[8] F. Peng et al., "Motion artifact removal from photoplethysmographic signals by combining temporally constrained independent component analysis and adaptive filter," BioMedical Engineering OnLine, vol. 13, no. 1, p. 50, 2014

[9] K. A. Reddy et al., "Use of Fourier series analysis for motion artifact reduction and data compression of photoplethysmographic signals,' IEEE Trans. Instrum. Meas., vol. 58, no. 5, pp. 1706-1711, May 2009.

[10] S. M. López Silva et al., "Heuristic algorithm for photoplethysmographic heart rate tracking during maximal exercise test," J. Medical and Biological Engineering, vol. 32, no. 3, pp. 181-188, 2012

[11] H. Fukushima et al., "Estimating heart rate using wrist-type photoplethysmography and acceleration sensor while running," in Proc. Ann. Int. Conf. IEEE Eng. Med. Biol. Society (EMBC), 2012, pp. 2901-2904.

[12] B. Sun and Z. Zhang, "Photoplethysmography-based heart rate monitoring using asymmetric least squares spectrum subtraction and Bayesian decision theory," IEEE Sensors J., vol. 15, no. 12, pp. 7161-7168, Dec 2015.

[13] Z. Zhang, "Photoplethysmography-based heart rate monitoring in physical activities via joint sparse spectrum reconstruction," IEEE Trans. Biomed. Eng., vol. 62, no. 8, pp. 1902-1910, 2015.

[14] Y. Wang et al., "Heart rate monitoring from wrist-type PPG based on singular spectrum analysis with motion decision," in Proc. Ann. Int Conf. IEEE Eng. Med. Biol. Society (EMBC), Aug 2016, pp. 3511-3514.

[15] Z.-H. Zhang et al., "A new framework to extract heart rate information from photoplethysmographic (PPG) signals with strong motion artifacts," in Proc. IEEE Region 10 Conf. (TENCON), Nov 2015, pp. 1-4.

[16] T. Schäck et al., "A new method for heart rate monitoring during physical exercise using photoplethysmographic signals," in Proc. European Signal Process. Conf., Aug 2015, pp. 2666-2670.

[17] S. Zhu et al., "MICROST: A mixed approach for heart rate monitoring during intensive physical exercise using wrist-type PPG signals," in Proc. Ann. Int. Conf. IEEE Eng. Med. Biol. Society (EMBC), Aug 2015, pp. 2347-2350.

[18] F. Gustafsson et al., "Particle filters for positioning, navigation, and tracking," IEEE Trans. Signal Process., vol. 50, no. 2, pp. 425-437, 2002.

[19] M. S. Arulampalam et al., "A tutorial on particle filters for online nonlinear/non-Gaussian Bayesian tracking," IEEE Trans. Signal Process., vol. 50, no. 2, pp. 174-188, 2002.

[20] G. Kitagawa, "Monte Carlo filter and smoother for non-Gaussian nonlinear state space models," J. Comput. Graph. Stat., vol. 5, no. 1, pp. $1-25,1996$.

[21] J. Martin Bland and D. G. Altman, "Statistical methods for assessing agreement between two methods of clinical measurement," The Lancet, vol. 327, no. 8476, pp. 307-310, 1986

[22] Z. Zhang. (2016) MFOCUSS. [Online]. Available: https://sites.google. com/site/researchbyzhang/software

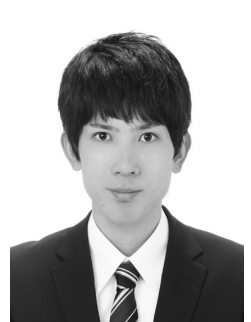

Yuya Fujita received his B.E. degree in Electrical and Electronic Engineering from Kyoto University in 2012, and is currently a master course student at Department of Communications and Computer Engineering, Kyoto University. His research interests include digital signal processing. He is a member of IEICE 


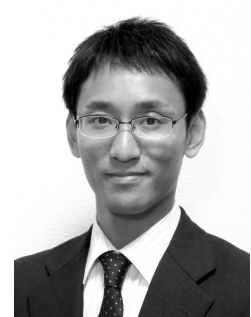

of IEICE and IPSJ.

Masayuki Hiromoto received B.E. degree in Electrical and Electronic Engineering and M.Sc. and Ph.D. degrees in Communications and Computer Engineering from Kyoto University in 2006, 2007, and 2009 respectively. He was a JSPS research fellow from 2009 to 2010, and with Panasonic Corp. from 2010 to 2013. In 2013, he joined the Graduate School of Informatics, Kyoto University, where he is currently an assistant professor. His research interests include VLSI design methodology, image processing and pattern recognition. He is a member

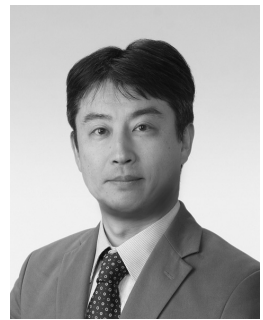

Takashi Sato received B.E. and M.E. degrees from Waseda University, Tokyo, Japan, and a Ph.D. degree from Kyoto University, Kyoto, Japan. He was with Hitachi, Ltd., Tokyo, Japan, from 1991 to 2003, with Renesas Technology Corp., Tokyo, Japan, from 2003 to 2006, and with the Tokyo Institute of Technology, Yokohama, Japan. In 2009, he joined the Graduate School of Informatics, Kyoto University, Kyoto, Japan, where he is currently a professor. He was a visiting industrial fellow at the University of California, Berkeley, from 1998 to 1999 . His research interests include CAD for nanometer-scale LSI design, fabrication-aware design methodology, and performance optimization for variation tolerance. Dr. Sato is a member of IEICE. He received the Beatrice Winner Award at ISSCC 2000. 